\title{
Avaliação eritrocitária de eqüinos hígidos submetidos a um protocolo hemodialítico*
}

\section{Erythrocytes evaluation in healthy horses submitted to a hemodialysis protocol}

\author{
Juliana de Oliveira, ${ }^{* *}$ Maristela Silveira Palhares, ${ }^{* * *}$ Mateus Teixeira Sampaio, ${ }^{* * *}$ Ubiratan Pereira Melo, ${ }^{* * * *}$ \\ Lucas Tadeu Silva, ${ }^{* * *}$ José Monteiro da Silva Filho***
}

\begin{abstract}
Resumo
Com o objetivo de realizar a adequação da técnica de hemodiálise para eqüinos, foram formados quatro grupos experimentais de seis animais cada, sendo determinados os seguintes tratamentos: Grupo I: animais sadios submetidos a cateterismo central unilateral e protocolo de sedação (grupo controle); Grupo II: animais sadios submetidos a cateterismo central unilateral com cateter duplo-lúmen e uma sessão de hemodiálise de seis horas; Grupo III: animais sadios submetidos a cateterismo central unilateral com cateter duplo-lúmen e duas sessões de hemodiálise de seis horas; Grupo IV: animais sadios submetidos a cateterismo central bilateral com cateter mono-lúmen e uma sessão de hemodiálise de seis horas. Empregou-se xilazina $10 \%(0,4 \mathrm{mg} / \mathrm{kg})$ associada a acepromazina $2 \%(0,08 \mathrm{mg} / \mathrm{kg})$ via intravenosa para sedação. Foram utilizados dois hemodialisadores dispostos em série, do tipo fibras ocas, baixo fluxo, membrana de polissulfona e área de $1,8 \mathrm{~m}^{2}$. $\mathrm{O}$ fluxo sanguíneo médio do circuito extracorpóreo foi de 319,18 $\pm 97,41 \mathrm{ml} /$ minuto. A anticoagulação foi feita com heparina sódica em $100 \mathrm{Ul} / \mathrm{kg}$ para primming, repetida na dose de 53,86 $\pm 18,61 \mathrm{Ul} / \mathrm{kg} / \mathrm{hora}$. Dentre as respostas pesquisadas neste estudo, na avaliação hematológica observou-se diminuição nos valores de eritrócitos e índices hematimétricos, sendo estes achados condizentes com o emprego de um circuito extracorpóreo. Concluiu-se que o procedimento de hemodiálise pode ser empregado na espécie eqüina, empregando-se a técnica clássica descrita neste trabalho. Contudo, a avaliação hematológica antes e depois de cada sessão de diálise é fundamental para monitorização do paciente.
\end{abstract}

Palavras-chave: eqüinos, hemodiálise, diálise, hematologia, eritrograma.

\begin{abstract}
With the goal of promote hemodialysis technical adequacy for horses, four experimental groups with six animals was formed, and the folowing treatments was applied: Group I: horses submited to unilateral central venous catheter and a sedation protocol (control group); Group II: horses submited to unilateral central venous double lumen catheter and one session of six hours hemodialysis; Group III: horses submited to unilateral central venous double lumen catheter and two sessions of six hours hemodialysis; Group IV: horses submited to bilateral central venous mono lumen catheter and one session of six hours hemodialysis. Xilazine $10 \%(0,1 \mathrm{ml} / 50 \mathrm{~kg})$ associated with acepromazine $2 \%(0,1 \mathrm{ml} / 50 \mathrm{~kg})$ was done by venous rote for sadation. Two hollow fiber, low flux polyssulfone of $1,8 \mathrm{~m}^{2}$ was used in serie connexion. The mean blood flux was $319,18 \pm 97,41$ $\mathrm{ml} / \mathrm{min}$. Antiocoagulation was performed with sodium heparin, $100 \mathrm{Ul} / \mathrm{kg}$ for primming and at the dose of 53,86 $\pm 18,61 \mathrm{Ul} / \mathrm{kg} / \mathrm{hr}$. For the evaluation performed in this study, in the blood analysis was observed decrease in eritrocites and hematimetric values. Was concluded that hemodialysis can be applied for horses by using the classical thecnic, described in this work, but, the blood evaluation before and after this procedure is fundamental for patients monnitoring.
\end{abstract}

Keywords: equine, hemodialysis, dialysis, hematology, eritrogram.

\section{Introdução}

Na espécie eqüina há poucos relatos de estudos com a técnica de hemodiálise (Vivrette et al., 1993; Ferreira et al., 2002; Guimarães et al., 2002). Entretanto, pesquisas já demonstraram a depuração de citocinas pró-inflamatórias
(TNF, IL-1 e IL-6) por meio da diálise sanguínea em eqüinos (Veenman et al., 2002), gerando grande expectativa em relação ao uso das terapias dialíticas em pacientes sépticos e endotoxêmicos. Nos eqüinos, muitas enfermidades têm origem infecciosa ou levam a sepse. Dentre estas doenças, as que cursam com a síndrome do abdômen agudo são

\footnotetext{
* Parte da tese da primeira autora - Doutorado em Ciência Animal - Escola de Veterinária da UFMG.

** Professora adjunto - Departamento de Medicina Veterinária, Universidade Estadual de Maringá. Bolsista CNPq durante doutoramento em Ciência Animal - EV - UFMG; juliana.deoliveira@yahoo.com.br

*** Professor Associado - Departamento de Clínica e Cirurgia Veterinárias, Escola de Veterinária UFMG.

${ }^{* * * *}$ Acadêmico de Medicina Veterinária - EV - UFMG.

*****Acadêmico do Curso de Doutorado em Medicina Veterinária - EV - UFMG.
} 
particularmente comuns na rotina de atendimento clínico, e estão invariavelmente associadas a septicemia ou endotoxemia e, comumente, ao óbito. Sob este aspecto, a aplicação da hemodiálise passa a ser um importante recurso no tratamento de eqüinos com síndrome cólica, dentre outras enfermidades (Veenman et al., 2002; Roy, 2004).

\section{Material e métodos}

Este trabalho experimental foi realizado nas instalações do Hospital Veterinário, na Clínica Médica de Eqüinos e Laboratório de Patologia Clínica da Escola de Veterinária da Universidade Federal de Minas Gerais, localizada no município de Belo Horizonte. O período experimental compreendeu o intervalo dos meses de abril a setembro de 2006.

Previamente à aquisição e transporte, os animais foram submetidos à sorologia para anemia infecciosa eqüina, cujos resultados não apontaram indivíduos positivos. Foram realizados exames clínico e hematológico (hemograma e pesquisa de hemoparasitas). Todos os animais receberam tratamento ectoparasiticida. As fêmeas foram avaliadas quanto à função reprodutiva por meio da palpação transretal, sendo diagnosticado quatro animais gestantes, em períodos gestacionais que variaram entre o terceiro e quinto mês de gestação.

O manejo nutricional utilizado durante todo o período experimental consistiu de fornecimento diário de capim elefante (Pennisetum purpureum) picado ad libitum, associado a cerca de $4 \mathrm{~kg} /$ animal de feno de Tifton (Cynodon sp.). Além destes, foi oferecida ração comercial ${ }^{1}$ para eqüinos adultos, uma vez ao dia, na quantidade de $1 \mathrm{~kg} / \mathrm{animal}$, sal mineral ${ }^{2}$ na quantidade de $100 \mathrm{~g} / \mathrm{animal}$, e água ad libitum. Os primeiros 90 dias foram considerados período de adaptação, no qual as éguas foram acompanhadas diariamente, e somente aquelas consideradas clinicamente sadias foram empregadas neste estudo.

Os animais foram, inicialmente, separados de acordo com o peso, escore corporal e estado reprodutivo (gestante ou não gestante). Em seguida, foram sorteados aleatoriamente para formação de quatro grupos experimentais de seis animais cada. As éguas do grupo I (controle) foram reutilizadas nos grupos II e IV, obedecendo a um intervalo mínimo de 21 dias. Os animais utilizados no grupo III foram os mesmos do grupo II, após 48 horas. Os tratamentos experimentais estão descritos na Tabela 1.

Para a contenção física dos animais foi utilizado cabresto e tronco de contenção para eqüinos. A contenção química foi efetuada pelo uso intravenoso de xilazina $10 \%{ }^{3}$ na dose de $0,4 \mathrm{mg} / \mathrm{kg}$ de peso corporal, associada a acepromazina $2 \%{ }^{4}$, na dose de $0,008 \mathrm{mg} / \mathrm{kg}$ de peso corporal. O procedimento de sedação precedeu o cateterismo em todos os grupos, e em alguns animais dos grupos II, III e IV houve a necessidade de repetição do protocolo sedativo após a terceira hora de diálise.

\footnotetext{
${ }^{1}$ Equitage 15P - Guabi Nutrição Animal - Brasil.

${ }^{2}$ Guabiphos Centauro 80 - Guabi Nutrição Animal - Brasil.

${ }^{3}$ Sedomin $^{\circledR}$, König, Brasil.

${ }^{4}$ Acepram 1,0\% ${ }^{\circledR}$, Univet, Brasil.
}

Em todos os grupos o cateterismo foi realizado após a sedação dos animais, e foi precedido de tricotomia ampla do sítio de punção, seguida pela anti-sepsia com solução de polivinilpirrolidona ${ }^{5}$ e álcool $70^{\circ}$. Todos os cateteres foram inseridos na veia jugular externa, no terço médio do pescoço, unilateralmente nos animais dos grupos I, II e III e bilateralmente nos animais do grupo IV. Após a introdução, a fixação externa do cateterismo foi realizada por meio de sutura na pele. Para o cateterismo dos animais do grupo I foi utilizado cateter de teflon ${ }^{6}$ de uso periférico, para acesso vascular temporário, mono-lúmen tamanho $14 \mathrm{G}$ e $4,8 \mathrm{~cm}$ de comprimento.

Para o cateterismo dos animais dos grupos II e III foram empregados cateteres de silicone ${ }^{7}$ de utilização central, para acesso vascular permanente, do tipo duplo-lúmen coaxial tamanho $13 \mathrm{Fr}$ e $30 \mathrm{~cm}$. Em função do diâmetro, estes cateteres foram inseridos após pequena ressecção da pele com auxílio de bisturi (aproximadamente $1 \mathrm{~cm}$ ).

O cateterismo dos animais do grupo IV foi realizado por meio de punção direta com o cateter sendo, porém, necessária pequena ressecção prévia da pele em função do diâmetro e material do cateter. Neste grupo foram empregados cateteres de teflon de uso periférico, para acesso vascular temporário, do tipo mono-lúmen tamanhos $10 \mathrm{G}$ e $7,6 \mathrm{~cm}$ para o acesso de saída de sangue, e $12 \mathrm{G}$ e $7,6 \mathrm{~cm}$ para o retorno venoso.

Tabela 1: Grupos experimentais

\section{Grupos experimentais}

\section{Grupo I Animais sadios submetidos a cateterismo central unilateral e protocolo de sedação (grupo controle). \\ Grupo II Animais sadios submetidos a cateterismo central unilateral e uma sessão de hemodiálise clássica de seis horas de duração (uma sessão - duplo-lúmen). \\ Grupo III Animais sadios submetidos a cateterismo central unilateral e duas sessões de hemodiálise clássica de seis horas de duração e intervalo interdialítico de 48 horas (duas sessões - duplo-lúmen). \\ Grupo IV Animais sadios submetidos a cateterismo central bilateral e uma sessão de hemodiálise clássica de seis horas de duração (uma sessão - mono-lúmen).}

Nos animais dos grupos II, III e IV, além do protocolo para tranqüilização optou-se pela realização de anestesia local no sítio de colocação do cateter, pela necessidade prévia da incisão da pele. O cateterismo foi mantido apenas o tempo requerido para realização do procedimento de hemodiálise.

A hemodiálise foi realizada em todos os animais dos grupos II, III e IV. A sessão de diálise foi iniciada 30 minutos após o procedimento de sedação, e teve duração de seis horas. O volume médio do fluxo sanguíneo foi de $319,18 \pm 97,41 \mathrm{ml} /$ minuto, acompanhado por fluxo de dialisato de $500 \mathrm{ml} /$ minuto. A máquina de hemodiálise utilizada foi uma proporcionadora

\footnotetext{
${ }^{5}$ Povidine ${ }^{\circledR}$, Riodeine, Brasil.

${ }^{6}$ Angiocath - BD, BD do Brasil.

7 Joline GmbH \& Co., Euromed Cateteres, Lagoa Santa.
} 
individual, modelo $2008-C,{ }^{8}$ acoplada a unidade portátil de tratamento de água, modelo WTU 100.

Para a formação do dialisato utilizaram-se as soluções concentradas padrões específicas para hemodiálise ácida e básica (bicarbonato). A composição de cada solução está descrita na Tabela 2.

O hemodialisador ${ }^{9}$ utilizado para todas as hemodiálises foi do tipo fibras ocas, de baixo fluxo, com membrana sintética de polissulfona e superfície de troca de $1,8 \mathrm{~m}^{2}$. Utilizaram-se linhas de sangue de tamanho adulto, ${ }^{11} \mathrm{com}$ volume de preenchimento de $70 \mathrm{ml}$ cada. Para cada diálise foram empregados dois hemodialisadores dispostos em série.

Para anticoagulação do circuito extracorpóreo empregou-se heparina sódica ${ }^{10}$ na dose de $100 \mathrm{UI} / \mathrm{kg}$ de peso corporal, para o procedimento de "primming" no início da diálise. A heparina foi repetida na dose de 1,33 $\pm 0,64 \mathrm{ml} / \mathrm{kg}$, a intervalos de 60 minutos, sendo estas aplicações interrompidas uma hora antes do término de cada hemodiálise. Após o término de cada sessão, todos os animais receberam uma dose de sulfato de protamina ${ }^{11}$ via venosa, equivalente em mililitros à última dose de heparina sódica utilizada.

Os animais permaneceram em posição ortostática durantes as seis horas de hemodiálise, e dentre as respostas obtidas foram avaliadas a contagem e morfologia eritrocitárias, sendo os dados anotados em fichas específicas.

Tabela 2: Composição básica das soluções concentradas para hemodiálise

\begin{tabular}{ccc}
\hline Composição química & Solução ácida & Solução base \\
\hline Sódio $(\mathrm{mEq} / \mathrm{l})$ & 138,0 & 138 \\
Potássio $(\mathrm{mEq} / \mathrm{l})$ & 3,5 & - \\
Cálcio $(\mathrm{mg} / \mathrm{dl})$ & 14 & - \\
Cloretos $(\mathrm{mEq} / \mathrm{l})$ & 109,5 & - \\
Magnésio $(\mathrm{mg} / \mathrm{dl})$ & 1,8 & 32,2 \\
Bicarbonato $(\mathrm{mEq} / \mathrm{l})$ & - & $6,8-7,2$ \\
$\mathrm{pH}$ & $6,8-7,2$ & $12,2-14,4$ \\
\hline Condutividade & $12,2-14,4$ & \\
\hline
\end{tabular}

Fonte: Fresenius Medical Care

Amostras de sangue foram coletadas em frasco contendo EDTA (sal dissódico do ácido etileno diamino tetra-acético) para a realização do hemograma, sendo o esfregaço sanguíneo realizado no momento da coleta. Para este esfregaço empregou-se posteriormente a coloração de MayGrünwald Giemsa (MGG), com o objetivo de se verificar a morfologia celular. Todos os hemogramas foram processados eletronicamente $^{12}$ ao final de cada sessão de hemodiálise.

\footnotetext{
${ }^{8}$ Fresenius Medical Care

${ }^{9}$ Fresenius Polysulfone ${ }^{\circledR}$ Capillary Dialysers - Hemoflow F8 - Series Low-Flux - resenius Medical Care

${ }^{10}$ Heparin - Cristalia Produtos Químicos Farmacêuticos Ltda.

11 Sulfato de protamina - ICN.

${ }^{12}$ Abacus Junior Vet - Hematology Analyser, Diatron.
}

O procedimento de coleta de sangue nos animais do grupo I foi feito por meio de punção direta da veia jugular externa, utilizando-se agulha de punção ${ }^{13}$ para tubo a vácuo. Nos indivíduos dos grupos II, III e IV o ponto de eleição para coleta de sangue foi a linha de hemodiálise, por esta possuir um dispositivo específico para este fim.

O delineamento experimental foi inteiramente ao acaso. $\mathrm{O}$ modelo constituiu-se de parcelas subdivididas (quatro grupos nas parcelas, e tempo de coleta de amostras e interações nas subparcelas, com seis repetições).

A análise de variância foi utilizada (PROC GLM), considerandose a ocorrência dos erros (a) e (b), referentes à parcela e subparcela, respectivamente. O teste estatístico utilizado para a comparação das médias obtidas foi o teste de Student Newman Keuls (SNK), com nível de significância de 95\% $(p<0,05)$.

\section{Resultados e discussão}

O acompanhamento hematológico de indivíduos em hemodiálise é importante, já que a passagem do sangue por um circuito extracorpóreo pode ocasionar diversas alterações sanguíneas. A avaliação hematológica deste estudo descreve os dados do eritrograma (eritrócitos, hematócrito, RDW, VCM, hemoglobina, CHCM e CHM) (Tabela 3).

Na observação da contagem eritrocitária, apesar das diferenças $(p<0,05)$ observadas, os valores de eritrócitos nos grupos I, II e IV estão dentro dos limites fisiológicos para a espécie eqüina. Exceção é feita aos animais do grupo III, que demonstraram valores abaixo da normalidade, nos tempos de 1 a 6 .

A diminuição na contagem eritrocitária ocorreu em todos os grupos, comparando-se a amostra 0 com as demais $(p<0,05)$. A diminuição inicial da contagem eritrocitária em todos os grupos é decorrente do uso de xilazina e acepromazina, como citam Courtot et al. (1978) e Dyke (1993).

Os resultados verificados para o hematócrito apresentaram resposta semelhante à contagem eritrocitária, observandose, apesar das diferenças numéricas, valores fisiológcos para a espécie eqüina nos grupos I, II e IV. Uma pequena diferença no grupo II, trata-se da amostra 6 deste grupo, com hematócrito abaixo dos valores esperados. Também a semelhança dos eritrócitos, a diminuição $(p<0,05)$ do volume globular é diagnosticada nos animais do grupo III, nos tempos de 1 a 6 .

Já os valores de RDW demonstraram diferenças $(p<0,05)$ entre as amostras e entre os grupos. A ausência de alterações acentuadas no RDW foi condizente com os achados de morfologia celular, avaliada por microscopia óptica, pela qual não foram detectadas alterações no tamanho dos eritrócitos, sendo todos os exames considerados normocíticos em todos os tempos.

Os indivíduos do grupo controle, não dialisado, apresentaram queda numérica dos valores de eritrócitos semelhante aos grupos II e IV, indicando influência de outro componente, que não somente a hemodiálise, sobre os resultados. Muir et al. (1979) destacaram as alterações hemodinâmicas causadas pela associação de acepromazina e xilazina em eqüinos, devido à diminuição do volume globular, e Fantoni et al. (1999)

${ }^{13}$ Vaccuette do Brasil 
Tabela 3: Avaliação do eritrograma em eqüinos hígidos submetidos à hemodiálise (média \pm desvio-padrão)

\begin{tabular}{|c|c|c|c|c|}
\hline $\begin{array}{c}\text { Grupos* } \\
\text { Tempos }^{\star \star}\end{array}$ & GI & GII & GIII & GIV \\
\hline \multicolumn{5}{|c|}{ Eritrócitos $\left(10^{6} / \mu \mathrm{l}\right)$} \\
\hline Amostra 0 & $7,24 \pm 1,12^{\mathrm{Aac}}$ & $8,05 \pm 0,75^{\mathrm{Ab}}$ & $6,70 \pm 1,33^{\mathrm{Aa}}$ & $7,37 \pm 0,95^{\mathrm{AC}}$ \\
\hline Amostra 1 & $6,15 \pm 0,92^{\mathrm{Ba}}$ & $6,26 \pm 0,86^{\mathrm{BCa}}$ & $5,34 \pm 1,60^{\mathrm{Bb}}$ & $5,86 \pm 0,57^{\mathrm{Bab}}$ \\
\hline Amostra 2 & $6,32 \pm 1,05^{\mathrm{Ba}}$ & $5,94 \pm 0,71^{\mathrm{Ba}}$ & $5,19 \pm 0,55^{\mathrm{Bb}}$ & $5,80 \pm 0,59^{\mathrm{Ba}}$ \\
\hline Amostra 3 & $6,43 \pm 1,06^{\mathrm{Ba}}$ & $6,18 \pm 0,74^{\mathrm{BDab}}$ & $5,14 \pm 1,28^{\mathrm{BCb}}$ & $5,95 \pm 0,70^{\mathrm{Ba}}$ \\
\hline Amostra 4 & $6,49 \pm 0,93^{\mathrm{Ba}}$ & $6,30 \pm 0,65^{\mathrm{BDa}}$ & $4,87 \pm 1,59^{\mathrm{BCb}}$ & $6,11 \pm 0,36^{\mathrm{Ba}}$ \\
\hline Amostra 5 & $6,44 \pm 1,08^{\mathrm{Ba}}$ & $6,68 \pm 0,61^{\mathrm{CDa}}$ & $4,71 \pm 1,78^{\mathrm{BCc}}$ & $6,32 \pm 0,58^{\mathrm{Ba}}$ \\
\hline Amostra 6 & $6,59 \pm 1,23^{\mathrm{Ba}}$ & $5,77 \pm 0,43^{\mathrm{Bb}}$ & $4,57 \pm 1,62^{\mathrm{Cc}}$ & $5,85 \pm 0,74^{\mathrm{Ba}}$ \\
\hline Amostra 7 & $6,60 \pm 0,79^{\mathrm{Ba}}$ & $6,91 \pm 1,40^{\mathrm{Cb}}$ & $6,39 \pm 0,66^{\mathrm{Aab}}$ & $6,25 \pm 1,00^{\mathrm{Ba}}$ \\
\hline \multicolumn{5}{|c|}{ Hematócrito (\%) } \\
\hline Amostra 0 & $31,28 \pm 4,52^{\mathrm{Aa}}$ & $31,68 \pm 1,28^{\mathrm{Aa}}$ & $26,32 \pm 5,10^{\mathrm{Ab}}$ & $30,42 \pm 4,04^{\mathrm{Aa}}$ \\
\hline Amostra 1 & $26,30 \pm 3,37^{\mathrm{Ba}}$ & $24,45 \pm 2,84^{\mathrm{Ba}}$ & $21,47 \pm 6,30^{\mathrm{Bb}}$ & $24,53 \pm 3,30^{\mathrm{Ba}}$ \\
\hline Amostra 2 & $26,81 \pm 3,51^{\mathrm{Ba}}$ & $23,32 \pm 2,15^{\mathrm{Bb}}$ & $20,89 \pm 5,93^{\mathrm{BCC}}$ & $24,28 \pm 3,23^{\mathrm{Bb}}$ \\
\hline Amostra 3 & $27,20 \pm 3,35^{\mathrm{Ba}}$ & $24,25 \pm 2,32^{\mathrm{BDb}}$ & $20,85 \pm 4,88^{\mathrm{BCc}}$ & $24,80 \pm 3,35^{\mathrm{Bb}}$ \\
\hline Amostra 4 & $27,55 \pm 3,35^{\mathrm{Ba}}$ & $25,05 \pm 2,38^{\mathrm{BCb}}$ & $19,86 \pm 6,18^{\mathrm{BCC}}$ & $25,92 \pm 2,59^{\mathrm{Bab}}$ \\
\hline Amostra 5 & $27,41 \pm 3,80^{\mathrm{Ba}}$ & $26,47 \pm 1,66^{\mathrm{CDb}}$ & $19,39 \pm 6,97^{\mathrm{BCC}}$ & $26,10 \pm 3,75^{\mathrm{Bab}}$ \\
\hline Amostra 6 & $28,23 \pm 4,98^{\mathrm{Ba}}$ & $23,23 \pm 1,52^{\mathrm{Bb}}$ & $18,90 \pm 6,35^{\mathrm{Cc}}$ & $25,53 \pm 3,88^{\mathrm{Bd}}$ \\
\hline Amostra 7 & $28,10 \pm 2,96^{\mathrm{Ba}}$ & $27,02 \pm 5,43^{\mathrm{Cab}}$ & $24,85 \pm 3,20^{\mathrm{Ab}}$ & $26,38 \pm 6,05^{\mathrm{Bab}}$ \\
\hline \multicolumn{5}{|c|}{ Índice de anisocitose (RDW) (\%) } \\
\hline Amostra 0 & $19,08 \pm 0,85^{\mathrm{a}}$ & $20,22 \pm 0,54^{\mathrm{Ab}}$ & $20,13 \pm 0,41^{\mathrm{Bb}}$ & $19,45 \pm 0,85^{\text {Aa }}$ \\
\hline Amostra 1 & $19,03 \pm 0,81^{\mathrm{a}}$ & $20,00 \pm 0,65^{\mathrm{ABb}}$ & $20,00 \pm 0,56^{\mathrm{ABb}}$ & $19,35 \pm 0,83^{\mathrm{Aa}}$ \\
\hline Amostra 2 & $19,03 \pm 0,68^{a}$ & $19,93 \pm 0,60^{\mathrm{ABb}}$ & $19,72 \pm 0,51^{\mathrm{Bb}}$ & $19,43 \pm 1,01^{\mathrm{Ac}}$ \\
\hline Amostra 3 & $18,93 \pm 0,80^{a}$ & $19,80 \pm 0,47^{\mathrm{Bb}}$ & $19,95 \pm 0,43^{\mathrm{ABb}}$ & $19,22 \pm 1,04^{\mathrm{Aa}}$ \\
\hline Amostra 4 & $19,12 \pm 0,95^{\mathrm{a}}$ & $20,01 \pm 0,31^{\mathrm{ABb}}$ & $19,84 \pm 0,49^{\mathrm{ABb}}$ & $19,38 \pm 0,92^{\mathrm{Aa}}$ \\
\hline Amostra 5 & $19,00 \pm 0,83^{a}$ & $19,97 \pm 0,59^{\mathrm{ABb}}$ & $20,06 \pm 0,41^{\mathrm{Ab}}$ & $19,34 \pm 0,95^{\mathrm{Aa}}$ \\
\hline Amostra 6 & $19,15 \pm 0,91^{a}$ & $19,88 \pm 0,39^{\mathrm{ABb}}$ & $19,85 \pm 0,22^{\mathrm{ABb}}$ & $19,45 \pm 1,15^{\mathrm{Aa}}$ \\
\hline Amostra 7 & $18,85 \pm 0,7^{\mathrm{a}}$ & $19,87 \pm 0,45^{\mathrm{ABb}}$ & $19,90 \pm 0,29^{\mathrm{ABb}}$ & $19,88 \pm 0,83^{\mathrm{Bb}}$ \\
\hline \multicolumn{5}{|c|}{ VCM (fl) } \\
\hline Amostra 0 & $43,50 \pm 4,32^{a}$ & $39,50 \pm 2,59^{\mathrm{ABa}}$ & $39,33 \pm 2,50^{\mathrm{Ab}}$ & $41,50 \pm 4,85^{\mathrm{AC}}$ \\
\hline Amostra 1 & $43,33 \pm 3,83^{a}$ & $39,33 \pm 2,16^{\mathrm{Ab}}$ & $40,50 \pm 1,87^{\mathrm{Bc}}$ & $42,00 \pm 4,33^{\mathrm{ABd}}$ \\
\hline Amostra 2 & $42,83 \pm 4,17^{a}$ & $39,500 \pm 2,26^{\mathrm{ABb}}$ & $40,83 \pm 1,83^{\mathrm{Bc}}$ & $42,00 \pm 4,86^{\mathrm{ABa}}$ \\
\hline Amostra 3 & $42,83 \pm 4,07^{\mathrm{a}}$ & $39,50 \pm 2,07^{\mathrm{ABb}}$ & $41,00 \pm 2,19^{\mathrm{BCc}}$ & $41,83 \pm 5,04^{\mathrm{ABC}}$ \\
\hline Amostra 4 & $42,67 \pm 3,93^{a}$ & $40,00 \pm 1,79^{\mathrm{ABb}}$ & $41,20 \pm 2,68^{\mathrm{BCc}}$ & $42,67 \pm 4,84^{\mathrm{Ba}}$ \\
\hline Amostra 5 & $42,67 \pm 3,72^{\mathrm{a}}$ & $39,67 \pm 2,06^{\mathrm{ABb}}$ & $41,80 \pm 2,49^{\mathrm{Ca}}$ & $41,60 \pm 4,93^{\mathrm{ABa}}$ \\
\hline Amostra 6 & $43,00 \pm 3,90^{a}$ & $40,33 \pm 1,97^{\mathrm{Bb}}$ & $41,83 \pm 2,14^{\mathrm{Cc}}$ & $43,67 \pm 3,93^{\mathrm{Ca}}$ \\
\hline Amostra 7 & $42,67 \pm 3,93^{\mathrm{a}}$ & $39,33 \pm 2,50^{\mathrm{Ab}}$ & $38,80 \pm 2,94^{\mathrm{Ab}}$ & $42,33 \pm 5,43^{\mathrm{ABa}}$ \\
\hline \multicolumn{5}{|c|}{ Hemoglobina (g/dl) } \\
\hline Amostra 0 & $12,45 \pm 1,78^{\mathrm{Aa}}$ & $12,50 \pm 1,19^{\text {Aa }}$ & $10,43 \pm 1,90^{\mathrm{Ab}}$ & $12,37 \pm 1,29^{A a}$ \\
\hline Amostra 1 & $10,95 \pm 1,49^{\mathrm{Ba}}$ & $9,93 \pm 1,16^{\mathrm{BCbc}}$ & $9,11 \pm 2,42^{\mathrm{Bb}}$ & $10,08 \pm 1,20^{\mathrm{BC}}$ \\
\hline Amostra 2 & $11,13 \pm 1,26^{\mathrm{BCa}}$ & $9,52 \pm 1,10^{\mathrm{Bbc}}$ & $9,02 \pm 2,33^{\mathrm{Bb}}$ & $10,10 \pm 1,20^{\mathrm{Bc}}$ \\
\hline Amostra 3 & $11,55 \pm 1,59^{\mathrm{BCa}}$ & $9,92 \pm 1,14^{\mathrm{BCb}}$ & $9,05 \pm 1,97^{\mathrm{Bc}}$ & $10,28 \pm 1,28^{\mathrm{Bb}}$ \\
\hline Amostra 4 & $11,63 \pm 1,61^{\mathrm{ABa}}$ & $10,30 \pm 1,00^{\mathrm{BDb}}$ & $8,92 \pm 2,44^{\mathrm{Bc}}$ & $10,88 \pm 0,88^{\mathrm{Bab}}$ \\
\hline Amostra 5 & $11,45 \pm 1,56^{\mathrm{BCa}}$ & $10,68 \pm 0,75^{\mathrm{CDa}}$ & $8,70 \pm 2,62^{\mathrm{Bb}}$ & $10,88 \pm 1,42^{\mathrm{Ba}}$ \\
\hline Amostra 6 & $11,88 \pm 2,10^{\mathrm{ACa}}$ & $9,67 \pm 0,96^{\mathrm{Bb}}$ & $8,47 \pm 2,20^{\mathrm{BC}}$ & $10,78 \pm 1,60^{\mathrm{Bd}}$ \\
\hline Amostra 7 & $12,03 \pm 1,43^{\mathrm{ACa}}$ & $10,98 \pm 2,13^{\mathrm{Db}}$ & $10,07 \pm 1,37^{A c}$ & $10,45 \pm 1,53^{\mathrm{Bbc}}$ \\
\hline \multicolumn{5}{|c|}{ CHCM (\%) } \\
\hline Amostra 0 & $39,77 \pm 2,90^{A}$ & $39,33 \pm 2,44^{\mathrm{A}}$ & $39,65 \pm 2,55^{A}$ & $40,77 \pm 1,98^{\mathrm{AB}}$ \\
\hline Amostra 1 & $41,500 \pm 1,22^{\mathrm{ABab}}$ & $40,63 \pm 2,11^{\mathrm{ABa}}$ & $43,23 \pm 4,47^{\mathrm{Bb}}$ & $41,08 \pm 1,82^{\text {ABab }}$ \\
\hline Amostra 2 & $41,52 \pm 1,99^{\mathrm{ABa}}$ & $40,88 \pm 3,11^{\mathrm{ABa}}$ & $43,83 \pm 5,00^{\mathrm{BDb}}$ & $41,62 \pm 1,97^{\mathrm{ABa}}$ \\
\hline Amostra 3 & $42,35 \pm 2,26^{\mathrm{BAb}}$ & $40,88 \pm 2,00^{\mathrm{ABa}}$ & $43,72 \pm 3,76^{\mathrm{BDb}}$ & $41,52 \pm 1,16^{\mathrm{ABa}}$ \\
\hline Amostra 4 & $42,07 \pm 1,79^{\mathrm{Ba}}$ & $41,10 \pm 2,60^{\mathrm{ABa}}$ & $46,12 \pm 6,15^{\mathrm{BCb}}$ & $41,98 \pm 1,46^{\mathrm{ABa}}$ \\
\hline Amostra 5 & $41,73 \pm 0,87^{\mathrm{ABa}}$ & $40,40 \pm 3,39^{\mathrm{ABa}}$ & $46,54 \pm 7,17^{\mathrm{CDb}}$ & $41,66 \pm 1,30^{\mathrm{ABa}}$ \\
\hline Amostra 6 & $40,97 \pm 3,45^{\mathrm{ABa}}$ & $41,62 \pm 3,51^{\mathrm{Ba}}$ & $46,97 \pm 8,69^{\mathrm{Cb}}$ & $42,32 \pm 1,87^{\mathrm{Aa}}$ \\
\hline Amostra 7 & $42,77 \pm 1,01^{\mathrm{Ba}}$ & $40,75 \pm 1,47^{\mathrm{ABab}}$ & $39,90 \pm 2,52^{\mathrm{Ab}}$ & $40,11 \pm 3,18^{\mathrm{Bb}}$ \\
\hline \multicolumn{5}{|c|}{ HCM (pg) } \\
\hline Amostra 0 & $17,28 \pm 2,01^{\mathrm{a}}$ & $15,53 \pm 0,78^{\mathrm{Ab}}$ & $15,62 \pm 1,07^{\mathrm{Ab}}$ & $16,88 \pm 1,39^{\mathrm{Aa}}$ \\
\hline Amostra 1 & $17,88 \pm 1,92^{\mathrm{a}}$ & $15,93 \pm 0,90^{\mathrm{ABb}}$ & $17,48 \pm 2,08^{\mathrm{Ba}}$ & $17,20 \pm 1,42^{\mathrm{Aa}}$ \\
\hline Amostra 2 & $17,81 \pm 2,21^{\mathrm{a}}$ & $16,08 \pm 1,21^{\mathrm{ABb}}$ & $17,83 \pm 2,30^{\mathrm{BDa}}$ & $17,40 \pm 1,34^{\mathrm{Aa}}$ \\
\hline Amostra 3 & $18,10 \pm 1,75^{\mathrm{a}}$ & $16,12 \pm 1,05^{\mathrm{ABb}}$ & $17,88 \pm 1,73^{\mathrm{BDa}}$ & $17,33 \pm 1,79^{\mathrm{Aa}}$ \\
\hline Amostra 4 & $18,02 \pm 2,04^{a}$ & $16,37 \pm 0,83^{\mathrm{ABb}}$ & $18,96 \pm 2,74^{\mathrm{CDa}}$ & $17,83+1,58^{\mathrm{ABa}}$ \\
\hline Amostra 5 & $17,90 \pm 1,87^{\mathrm{a}}$ & $16,06 \pm 1,15^{\mathrm{ABb}}$ & $19,52 \pm 3,72^{\mathrm{Cc}}$ & $17,20 \pm 1,77^{\mathrm{ABa}}$ \\
\hline Amostra 6 & $17,68 \pm 2,10^{\mathrm{a}}$ & $16,77 \pm 2,10^{\mathrm{Bb}}$ & $19,72 \pm 4,17^{\mathrm{Cc}}$ & $18,53 \pm 2,81^{\mathrm{Ba}}$ \\
\hline Amostra 7 & $18,30 \pm 1,57^{\mathrm{a}}$ & $15,95 \pm 0,80^{\mathrm{ABbc}}$ & $15,50 \pm 0,81^{\mathrm{Ab}}$ & $16,83 \pm 1,40^{\mathrm{Ac}}$ \\
\hline
\end{tabular}

${ }^{A B}$ médias seguidas por letras maiúsculas, na mesma coluna, diferem $(P<0,05)$. ab médias seguidas por letras minúsculas, na mesma linha, diferem $(\mathrm{P}<0,05)$. ${ }^{* *}$ Amostra 0 : antes do início da hemodiálise; Amostra 1: 30 minutos após o início da diálise; Amostra 2: 60 minutos após o início da diálise; Amostra 3: 120 minutos após o início da diálise; Amostra 4: 210 minutos após o início da diálise; Amostra 5: 300 minutos após o início da diálise; Amostra 6: 15 minutos após o término da diálise = 375 minutos; Amostra 7: 24 horas após a hemodiálise. 
citam vasodilatação esplênica após a aplicação de acepromazina.

Entretanto, para o grupo III observa-se a queda dos valores eritrocitários abaixo do limite inferior. O grupo III se constitui dos mesmos animais do grupo II que foram redialisados após 48 horas da primeira diálise. Portanto, os animais do grupo III iniciaram este procedimento já com valores inferiores da contagem de eritrócitos, diferindo $(p<0,05)$ dos grupos II e IV. $\mathrm{O}$ efeito de diminuição na contagem de eritrócitos, somado a nova aplicação de sedativo e retomado do protocolo de coleta de amostras, pode ter proporcionado quedas mais acentuadas nos parâmetros avaliados.

Durante a diálise, além das coletas sanguíneas para análises, pode ocorrer perda de sangue durante 0 procedimento de lavagem do lúmen do cateter e na remoção de bolhas de ar do cata-bolhas. Estas perdas isoladas são insignificantes se comparadas ao volume de sangue dos eqüinos. Ao se somarem todos estes eventos ao uso de xilazina e acepromazina, e a repetição da hemodiálise no grupo III, parece provável que a redução na contagem eritrocitária e hematócrito tenha diversas etiologias.

Várias diferenças $(p<0,05)$ foram apontadas para os valores de VCM, porém, os resultados estão dentro dos limites fisiológicos. Estas observações estão de acordo com os valores do índice de anisocitose e com as observações realizadas a partir do esfregaço sanguíneo, onde não foram diagnosticadas alterações no tamanho dos eritrócitos. Estes achados concordam com as citações de Fishbane e Paganini (2003), que relataram ausência de alterações na morfologia eritrocitária, causada por diálise.

Na observação dos dados de hemoglobina detectaram-se diferenças $(p<0,05)$ entre os tempos de coleta, e entre os grupos nestes tempos. Os resultados demonstrados para a concentração de hemoglobina apresentaram resposta

\section{Agradecimentos}

Fresenius Medical Care e Euromed Cateteres.

\section{Referências}

COURTOT, D.; et al. The effect of acetylpromazine medication on red blood cell metabolism in the horse. Ann. Rech Veterinary, v. 9, n. 1, p. 17-24, 1978.

DYKE, T.M. Sedatives, tranquilizers, and stimulants. Vet. Clin. North Am.: Equine Practice, v. 9, n. 3, p. 621-634, 1993.

FANTONI, D.T.; et al. Avaliação comparativa entre a acepromazina, detomidina e romifidina em eqüinos. Ciência Rural, v. 29, n. 1, p. 4550, 1999.

FERREIRA, P. C. C.; et al. Utilização de hemodiálise em eqüinos endotoxêmicos. Relato de caso. Simpósio de nefrologia veterinária. Anais. p.65-70. 2002.

FISHBANE, S.; PAGANINI, E. Anormalidades hematológicas. In: DAUGIRDAS, J. T.; BLAKE, P. G. ING, T. S. Manual de diálise. Rio de Janeiro: Medsi, 2003. p. 491-508. semelhante àquela anteriormente citada para o VCM, onde, apesar das diferenças apontadas, nenhum valor em todos os grupos extrapolou os limites preconizados para a concentração de hemoglobina. A diminuição na concentração de hemoglobina acompanhou a diminuição apresentada para a contagem eritrocitária; contudo, nesta última, o decréscimo observado foi em menor proporção. A este fato está atribuído à hemólise durante o circuito extracorpóreo e o estresse oxidativo sobre os eritrócitos, como descrito por Lucchi et al. (2000), sendo a hemoglobina recuperada.

A elevação observada para a concentração de hemoglobina corpuscular média (CHCM) foi causada pela diminuição, proporcionalmente menor na hemoglobina, em relação aos eritrócitos, em função deste parâmetro ser calculado a partir dos valores de hemoglobina e hematócrito. Analisando estes dados conjuntamente com as respostas obtidas para a hemoglobina corpuscular média, observou-se que a regularidade nos valores desta medida foi mantida, não havendo valores abaixo ou superiores àqueles tomados como limites de referência.

A avaliação conjunta da contagem eritrocitária e dos índices hematimétricos sugerem um maior efeito da sedação sobre estes parâmetros, sendo observado diminuição dos valores iniciais em todos os grupos. Ocorreu também efeito da hemodiálise, principalmente sobre o decréscimo na contagem eritrocitária e hematócrito, como observado no grupo III.

\section{Conclusões}

A hemodiálise ocasiona diminuição da contagem eritrocitária, volume globular e hemoglobina, sem entretanto, ocasionar alterações hematológicas significativas.
GUIMARÃES, P. T. C.; et al. Hemodiálise em eqüino: relato de caso. Simpósio de nefrologia veterinária. Anais. p. 71-74. 2002.

LUCCHI, L.; et al. Artificial Organs, v. 24, n. 1, p. 1-6, 2000.

MUIR, W. W.; et al. Hemodynamic and respiratory effects of a xilazineacetylpromazine drug combination in horses. Am. J. Vet. Res., v. 40, n. 11, p. 1518-1522, 1979.

ROY, M. F. Sepse in adults and foals. Vet. Clin. - Equine Pract., v. 20, p. 41-61, 2004.

VEENMAN, J.N.; et al. High volume continuous venovenous haemofiltration (HV-CVVH) in an equine endotoxaemic shock model. Equ. Vet. J., v. 34, n. 5, p. 516-522, 2002.

VIVRETTE, S.; et al. Hemodialysis for treatment of oxytetracyclineinduced acute renal failure neonatal foal. J. of Am. Vet. Med. Ass., v. 203, n. 1, p. 105-107, 1993. 